\title{
Enhanced Rotational Feature Points Matching Using Orientation Correction
}

\author{
Kam Meng Goh, Syed Abd. Rahman Abu-Bakar, Musa Mohd. Mokji, Usman U. Sheikh \\ Department of Electronics and Computer Engineering, \\ Faculty of Electrical Engineering, \\ Universiti Teknologi Malaysia, \\ 81310, Skudai, Johor, Malaysia
}

Received 23rd Sep 2013; accepted 10 July 2014

\begin{abstract}
In matching between images, several techniques have been developed particularly for estimating orientation assignment in order to make feature points invariant to rotation. However, imperfect estimation of the orientation assignment may lead to feature mismatching and a low number of correctly matched points. Additionally, several possible candidates with high correlation values for one feature in the reference image may lead to matching confusion. In this paper, we propose a post-processing matching technique that will not only increase the number of correctly matched points but also manage to solve the above mentioned two issues. The key idea is to modify feature orientation based on the relative rotational degree between two images, obtained by taking the difference between the major correctly matched points in the first matching cycle. From the analysis, our proposed method shows that the number of detected points correctly matched with the reference image can be increased by up to $50 \%$. In addition, some mismatched points due to similar correlation values in the first matching round can be corrected. Another advantage of the proposed algorithm it that it can be applied to other state-of-the-art orientation assignment techniques.
\end{abstract}

Key Words: Orientation, Descriptor, Matching, Rotation

\section{Introduction}

In object detection and recognition, feature matching plays an important role in identifying similar objects seen from different views or angles. Some of the applications that use feature matching techniques are image stitching [1], fingerprint verification [2], tracking [3] and visual surveillance [4]. In order to reliably identify an object, the selected features must be robust to scale variance, noise, rotation, viewpoint changes and illumination changes. Typically, an object of interest in an image will go through feature extraction, scale-space construction, orientation assignment and descriptor generation before the feature matching process is applied. Among these stages, orientation assignment ensures that these features are rotationally invariant. If the features' orientation is incorrect, the matching process between the features may result in low correlation, which in return causes a high number of mismatches resulting in poor matching performance and a low number of correctly matched points. Hence, the number of correctly matched points is strongly

Correspondence to: <kenneth86gkm@gmail.com>

Recommended for acceptance by $<$ Giorgio Fumera $>$

ELCVIA ISSN: 1577-5097

Published by Computer Vision Center / Universitat Autonoma de Barcelona, Barcelona, Spain 
related to the orientation assignment. To make the feature rotationally invariant, various methods have been proposed, including Gabor filter (GB) [5], gradient magnitude with voting system (GV) [6], moment (MM) [7], gradient magnitude with hierarchy level (GH) [8] and Haar wavelet gradient extraction [9].

Most of the available state-of-the-art methods, however, focus on improving the computation of the orientation based on the gradient information. One such method is by using Gabor filters to determine feature point orientation. The Gabor filter is usually used to classify and describe texture $[5][10][11]$ as well as other applications such as fingerprint verification [12], document analysis [13] and retinal identification [14]. In [15], a few kernels were generated to check the orientation of the Gabor filter. They found that the accuracy was poor since the orientation generated was limited to eight directions only. In a different approach, image moments, proposed by H. Ming-Kuei [16], with their properties of scale and rotational invariance, were utilized for computing the orientation [17]. Some other well-known moments include Zernike moments [18], geometric moments [19] and complex moments [20]. However, the orientation calculated from these moments is easily influenced by noise. Rao presented a method to estimate the orientation of every pixel in fingerprint images based on gradient information [8]. However, noise in the image would affect the accuracy of the computed orientation. In [21], the step was further extended to obtain better results by applying a low pass Gaussian filter to smooth the orientation map so that it could be more resistant towards noise. In [22], hierarchical implementation was applied to extend the work by scaling down the image if the image variance was over the threshold value before estimating the orientation. However, selecting a threshold value is a difficult task. Similarly, in [6], the orientation was calculated using the gradient magnitude of the feature points and a voting system. Although the computed orientation could cope with noise, the method also produced multiple orientations and caused matching to perform poorly. The Haar wavelet based method was proposed in [9]. While this method is computationally faster compared to the one in [6], it may yield poor approximation as the parameter was chosen experimentally.

Based on the discussion above, we conclude that all of the above mentioned methods suffer from two major problems. First, imperfect estimation of the orientation computation leads to false matching if the corresponding candidate is disturbed by noise or illumination changes. This is because the orientation is computed based on the visual appearance information (i.e., vertical and horizontal gradient information) of the feature. Second, multiple corresponding candidates in the target image (with high correlation values) cause ambiguity which again contributes to incorrect matching These problems become even more evident if the input images share many similar features.

Hence, it is our goal in this paper to improve the matching rate while reducing the mismatch error by incorporating a new post-processing orientation correction method. We attempt to enhance the number of correctly matched points even with the presence of the mentioned limitations. The proposed method in this paper differs from existing techniques in that we do not address the orientation assignment stage, but rather enhancement after the matching process. Here, we assume that all features in the input image are rotated at a similar angle. The main advantages of our method are two-fold: 1) simplicity, and 2) portability, in the sense that the method can be applied to any feature matching process using state-of-the-art orientation assignment techniques. Basically, the proposed method consists of two steps: 1) estimation of the relative rotational angle between two images based on the matched points from the first matching cycle, 2) regeneration of new descriptor generation based on the estimated angle.

This paper is organized as follows. In Section 2, the general concept of the feature matching system is presented. In Section 3, the concept of the proposed technique is explained in detail, and the experimental comparisons among the state-of-the-art methods are shown in Section 4. Section 5 concludes the paper, highlighting the contributions of the proposed method. 


\section{Feature Extraction and Matching}

In matching the feature points, the two images basically need to go through four stages: feature point detection, orientation assignment, feature descriptor generation and matching, as illustrated in Fig. 1.

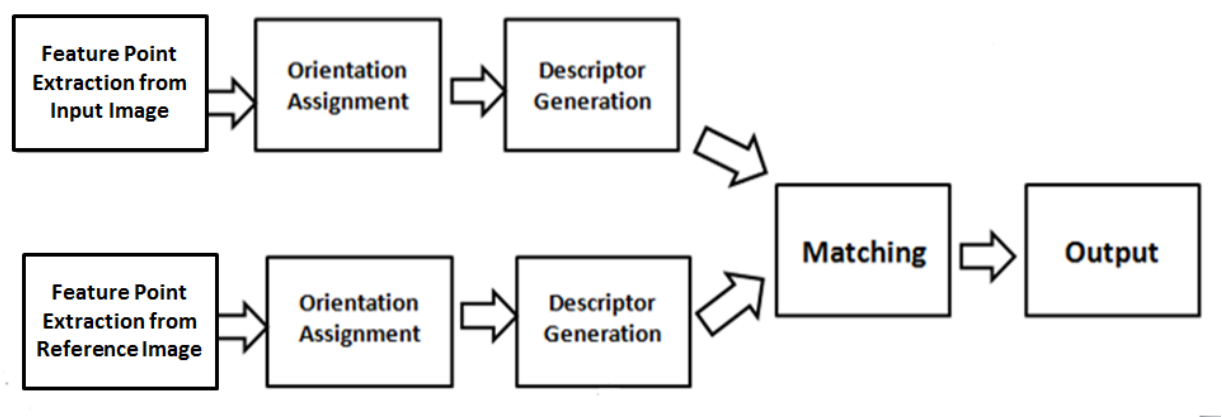

Fig. 1. Process of feature matching

\subsection{Feature Point Extraction}

This is the first step in which features are extracted from both images. In this paper, the Harris Corner detector is used to detect feature points [23]. Prewitt vertical and horizontal kernels are used to produce the gradient image, $I_{x}$ and $I_{y}$, respectively. Next, matrix $H$ is built for every pixel of the image based on $I_{x}$ and $I_{y}$. Subsequently, the determinant and the trace of $H$ are used to calculate the $C$ value for every pixel, which will build up a $C$ image. Then, local maximum suppression is applied using a kernel of size $3 \times 3$ on the $C$ image. $C(x, y)$ will be considered a point of interest if it has the highest value compared to the eight surrounding neighbours. Therefore, two sets of points from the input image, I, and reference image, R, are extracted respectively as denoted in Equations (1) and (2). $\mathrm{m}$ and $\mathrm{n}$ are the number of extracted points in each image. For further information on computing $H$ and $C$ refer to [9].

$$
\begin{aligned}
p_{I}= & \left\{\left(x_{I}, 1, y_{I, 1}\right),\left(x_{I, 2}, y_{I, 2}\right), \ldots,\left(x_{I, m}, y_{I, m}\right)\right\} \\
& p_{R}=\left\{\left(x_{R, 1}, y_{R, 1}\right),\left(x_{R, 2}, y_{R, 2}\right), \ldots,\left(x_{R, n}, y_{R, n}\right)\right\}
\end{aligned}
$$

\subsection{Orientation Assignment}

In this stage, we apply all the previously proposed methods described in Section 1 for the purpose of comparison. For the Gabor filter, a group of Gabor wavelets with different frequencies and different directions are normally computed. Then, the input image is convolved with all kernel magnitudes with different directions. Forty kernels are computed with five scales and eight orientations, $22.5^{\circ}, 45^{\circ}, 67.5^{\circ}, 90^{\circ}, 112.5^{\circ}, 135^{\circ}, 157.5^{\circ}$ and $180^{\circ}$, the same number of scales and orientations used in previous research [15]. The filtered image with the highest response will be considered as the orientation assignment. Fig. 2 shows eight kernels with different directions for one scale.

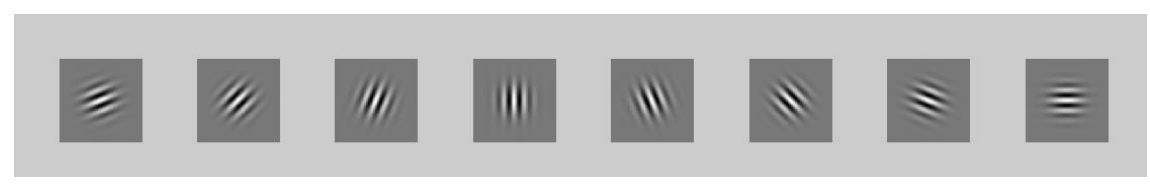

Fig. 2. Gabor filter with different directions on one scale. 
In Ming-Kuei's method, seven moment invariants including orientation were introduced. These seven moments are invariant to scaling, translation and rotation. Moments are weighted averages of a group of pixels of the object in an image. However, moments can only be applied to an image that is noise free. If the image is corrupted by noise, the moments may no longer be invariant to scale or rotation.

For gradient magnitude using the hierarchy level method, the image is first divided into even blocks of size $\mathrm{W} \times \mathrm{W}$. Then, vertical and horizontal gradients are calculated to compute the orientation and the Gaussian filter is applied for noise resistance purposes. After that, hierarchy implementation is applied, in which the variance of the orientation field is computed. If the value is above a certain threshold value, the local orientation of the subject pixel is re-estimated at a lower resolution until the variance is lower than the threshold value.

The calculation using gradient magnitude with a voting system is also based on gradient information. First, the input image is smoothed using a Gaussian filter. The gradient magnitude and the orientation of every pixel in a circular area of the Gaussian filter with a radius of 1.5 are calculated using pixel difference. To decide the orientation of the feature point, a voting system is used. An orientation histogram with 36 even bins is generated, with an interval of $10^{\circ}$ for each nonoverlapping bin. Each bin is built by adding the gradient magnitude of each sample and weighted with the Gaussian circular window if the sample is within the range of the bin. The bin with the highest value will be considered as the dominant orientation of the feature. If there are other bins which are within $80 \%$ of the highest bin, the feature may have multiple orientations. The multiple orientations may cause matching confusion.

By estimating the orientation using the Haar wavelet, first the region surrounding the feature is extracted. The Haar wavelet response in both the horizontal and vertical directions is computed in a circle of radius $6 \mathrm{~s}$, in which the circle is centred at the feature point and $\mathrm{s}$ is the current scale. Then, the responses are smoothed with a Gaussian circle with 2.5 s so that the responses become more stable. A segment covering $60^{\circ}$ is shifted every time for $10^{\circ}$ and all responses within the segment for each shifting are summed. The segment with the longest vector will be considered as the orientation area of the feature.

\subsection{Feature Descriptor Generation}

A variety of feature descriptors have been proposed in previous research, and among these are moment invariants [24], phase-based local features [25], steerable filters [26], or substantial amount and spatial intensity patterns of feature neighbourhoods [6][9]. Out of these descriptors, [6][9] outperform the others due to their descriptors being invariant to small deformations or localization errors. Therefore, we have adopted these techniques as our basic methods in our proposed method. With the calculated orientations of each feature point, descriptors of features are generated relative to the orientation. Initially, a square rotated region of size $20 \times 20$ centred at the feature point $(x, y)$ is extracted. The extracted region is divided into $4 \times 4$ sub-regions and each region contains $5 \times 5$ pixels. For each sub-region, the directional information, $d y$ and $d x$, and their absolute values are summed together to form four vectors. For the whole region, there will be $4 \times 4 \times 4$ vectors obtained with a vector length of 64 . The vector obtained is used as the descriptor of the feature and can be denoted as $D e_{m}=\left\{d_{m}, 1, d_{m}, 2, \ldots, d_{m}, 64\right\}$ and $D e_{n}=\left\{d_{n, 1}, d_{n, 2}, \ldots, d_{n, 64}\right\}$ for the input image and reference image respectively.

\subsection{Matching}

The most commonly used matching criteria is based on either the Euclidean distance or Mahalanobis distance. For instance, Euclidean distances of features are compared in [6][9]. The Euclidean distances of a pair of feature points will only be computed if a pair of features has the 
same type of contrast. However, as mentioned in Section 1, poor performance is expected due to ambiguity when several features with similar descriptor values exist for matching. In [27], a decision matrix is introduced whereby elements of the matrix are the correlation values between two features. Matching is undertaken by choosing the highest value in both row and column of the decision matrix. A similar concept is used in this paper but the matching is carried out by comparing the Euclidean distance of the descriptors of the features from two images. Letting $D(m, n)$ be a matrix of size $m \times n$ and all the elements inside this matrix be the Euclidean distances between descriptors as calculated using (3), the smallest value in both column and row will be considered as the matched pairs.

$$
D(m, n)=\sqrt{\sum_{i=1}^{64} D e_{m, i}-D e_{n, i}}
$$

\section{Proposed Method}

This paper proposes a method to improve the descriptor generation by providing orientation correction feedback from the matching process in order to generate refined orientations. This process is illustrated in Fig. 3. The following sub-sections describe the stages in achieving enhanced orientation correction. Since feature extraction, orientation assignment, and descriptor generation are widely outlined in previous works, we will not discuss these details further in this paper. Our focus is on the proposed post-processing stage.

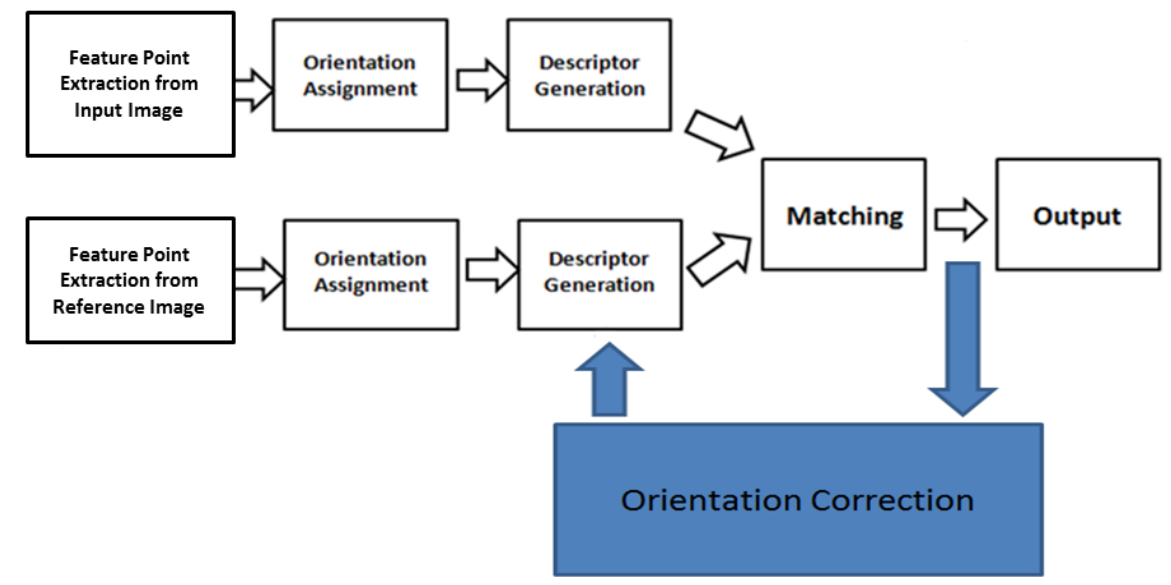

Fig. 3. Overall process of matching with extended technique for orientation correction

After the matching process, there will be a set of matched points which we denote as $\tilde{\boldsymbol{p}}$. However, some of the pairs in $\tilde{\boldsymbol{p}}$ may have false matched pairs due to more than one similar descriptor value. This section introduces a new technique to reduce these false matches and hence increase the number of correct matches using our proposed method of orientation correction at the postprocessing stage. The core idea of our method is based on the assumption that all features of the input image are rotated at a similar rotational angle if the input image undergoes a certain amount of fixed rotation. Even though it is simple, our proposed method not only removes the false matched points but, at the same time, increases the number of correctly matched points. Fig. 4 illustrates the details of the orientation correction strategy. Our proposed method consists of two important stages: first, the generation of the rotational angle and, second, the regeneration of new orientation assignments and descriptors for all features in the input image. 


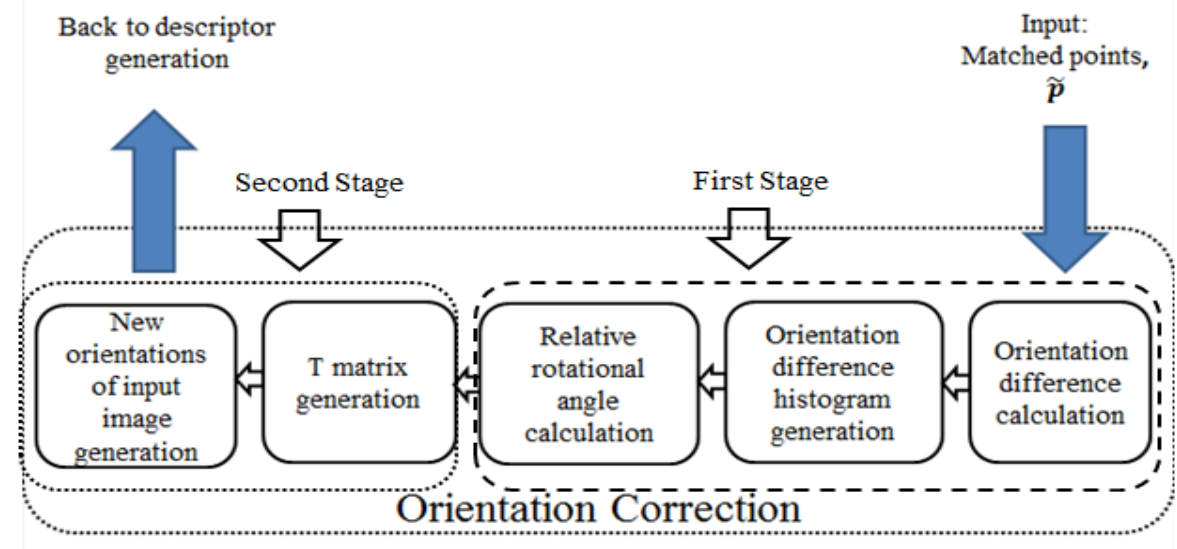

Fig. 4. Details of orientation correction strategy

\subsection{Relative Rotational Angle Generation}

Given a set of matched points, the orientation differences, $\theta_{d}$, of all matched pairs are computed using (4) where $\theta_{I} \mathrm{~s}$ are the orientations of the features from the input image while $\theta_{R} \mathrm{~s}$ are the orientations of the features from the reference image. $N=\left\{1,2,3, \ldots, p_{1}\right\}$, where $p_{1}$ is the number of matched pairs from the initial matching cycle.

$$
\theta_{d, N}=\theta_{I, N}-\theta_{R, N}
$$

Then, an orientation difference histogram from $0^{\circ}$ to $360^{\circ}$ with 36 bins and intervals of $10^{\circ}$ is built based on the orientation difference of all matched points, $\tilde{\boldsymbol{p}}$, while the heights of the bins represent the number of angles falling into that particular range of angles. The interval of the degree is chosen experimentally to achieve the best result. If most of the points are matched correctly, those matched pairs should have similar orientation differences, also called similar rotational angles. Fig. 5 shows an example of the histogram generated from a set of orientation differences with 15 data from Table 1 .

TABLE 1: EXAMPLE OF A SET OF ORIENTATION DIFFERENCES FROM THE MATCHED PAIRS.

\begin{tabular}{|l|l|l|l|l|l|l|l|l|l|l|l|l|l|l|l|}
\hline & $\theta_{d, 1}$ & $\theta_{d, 2}$ & $\theta_{d, 3}$ & $\theta_{d, 4}$ & $\theta_{d, 5}$ & $\theta_{d, 6}$ & $\theta_{d, 7}$ & $\theta_{d, 8}$ & $\theta_{d, 9}$ & $\theta_{d, 10}$ & $\theta_{d, 11}$ & $\theta_{d, 12}$ & $\theta_{d, 13}$ & $\theta_{d, 14}$ & $\theta_{d, 15}$ \\
\hline $\begin{array}{l}\text { Rotational } \\
\text { difference }\end{array}$ & $32^{\circ}$ & $37^{\circ}$ & $131^{\circ}$ & $41^{\circ}$ & $39^{\circ}$ & $11^{\circ}$ & $25^{\circ}$ & $35^{\circ}$ & $38.6^{\circ}$ & $134^{\circ}$ & $139^{\circ}$ & $37^{\circ}$ & $37^{\circ}$ & $31^{\circ}$ & $36^{\circ}$ \\
\hline
\end{tabular}




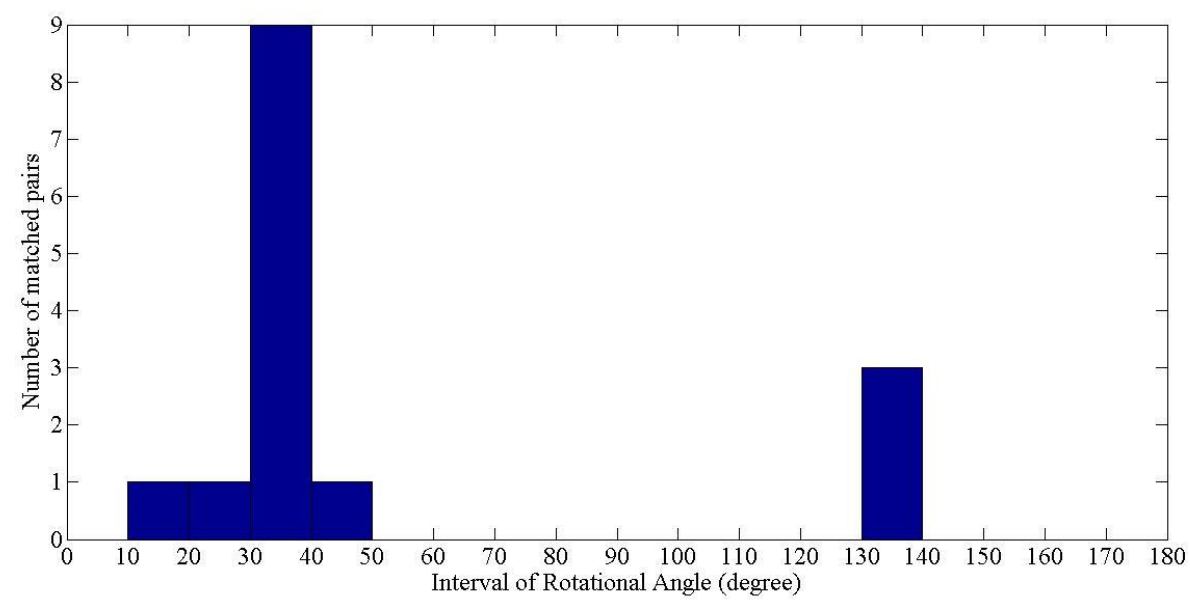

Fig. 5. Histogram of orientation differences generated

From the histogram, we can easily obtain the rotational angle between two images. We try to ascertain the relative rotational angle between two images based on the histogram. Since all features are assumed to be rotated at a similar angle or in the range of the angle, the highest bins of the histogram should represent the rotational degree between two images. For example, from Fig. 5 we know that the rotational angle is in the $3 \mathrm{rd}$ bin of the histogram, which is in the range 31 to $40^{\circ}$. Then, the relative rotational angle $\theta_{\text {avg }}$ is calculated by using (5), where $\theta_{h}$ is the orientation difference of each of the matched points in the range of the highest bin, $h$, and $p_{2}$ is the total number of angles in the range of the highest bin. In other words, the sum of all the angles falling into the highest range averaged by dividing the total value by the total number of angles that fall in the highest range. The samples which are not allocated to the highest bin will be considered as outliers.

$$
\theta_{\text {avg }}=\frac{\sum_{1}^{k=p_{2}} \theta_{h, k}}{p_{2}}
$$

\subsection{Updated Orientation and Descriptor Generation}

From the estimated relative rotational angles, we then generated a matrix of orientation differences, $T(x, y)$, of size $\mathrm{m} x \mathrm{n}$ as given in(6).

$$
T(m, n)=\left[\begin{array}{cccc}
\theta_{I, 1}-\theta_{R, 1} & \theta_{I, 2}-\theta_{R, 1} & \ldots & \theta_{I, n}-\theta_{R, 1} \\
\theta_{I, 1}-\theta_{R, 2} & \theta_{I, 2}-\theta_{R, 2} & \ldots & \theta_{I, n}-\theta_{R, 2} \\
\vdots & \vdots & \ddots & \vdots \\
\theta_{I, 1}-\theta_{R, m} & \theta_{I, 2}-\theta_{R, m} & \ldots & \theta_{I, n}-\theta_{R, m}
\end{array}\right]
$$

Then, all elements of $T$ were scanned and replaced by $\theta_{\text {avg }}$ if the particular angle is outside the range of $\theta_{\text {avg }} \pm 10^{\circ}$. With the updated values, the new dominant orientations for the descriptors of the features which are outside the range of $\theta_{a v g} \pm 10^{\circ}$ are regenerated using (7) by adding the relative rotational angle to the orientations of the features from the reference image.

$$
\theta^{\prime}{ }_{I}=\theta_{\text {avg }}+\theta_{R}
$$


The feature descriptors of the input image relative to the new orientations are generated again and a new matrix D based on the updated descriptor is computed. The matching process is then repeated based on the new matrix $D$ by choosing the minimum value in both the row and the column of the decision matrix. With these new regenerated descriptors, the falsely matched pairs will be corrected as illustrated in Fig. 6. Note that the right image in Fig. 6 is the zoomed version of Fig. 8(b) for better visual perception.

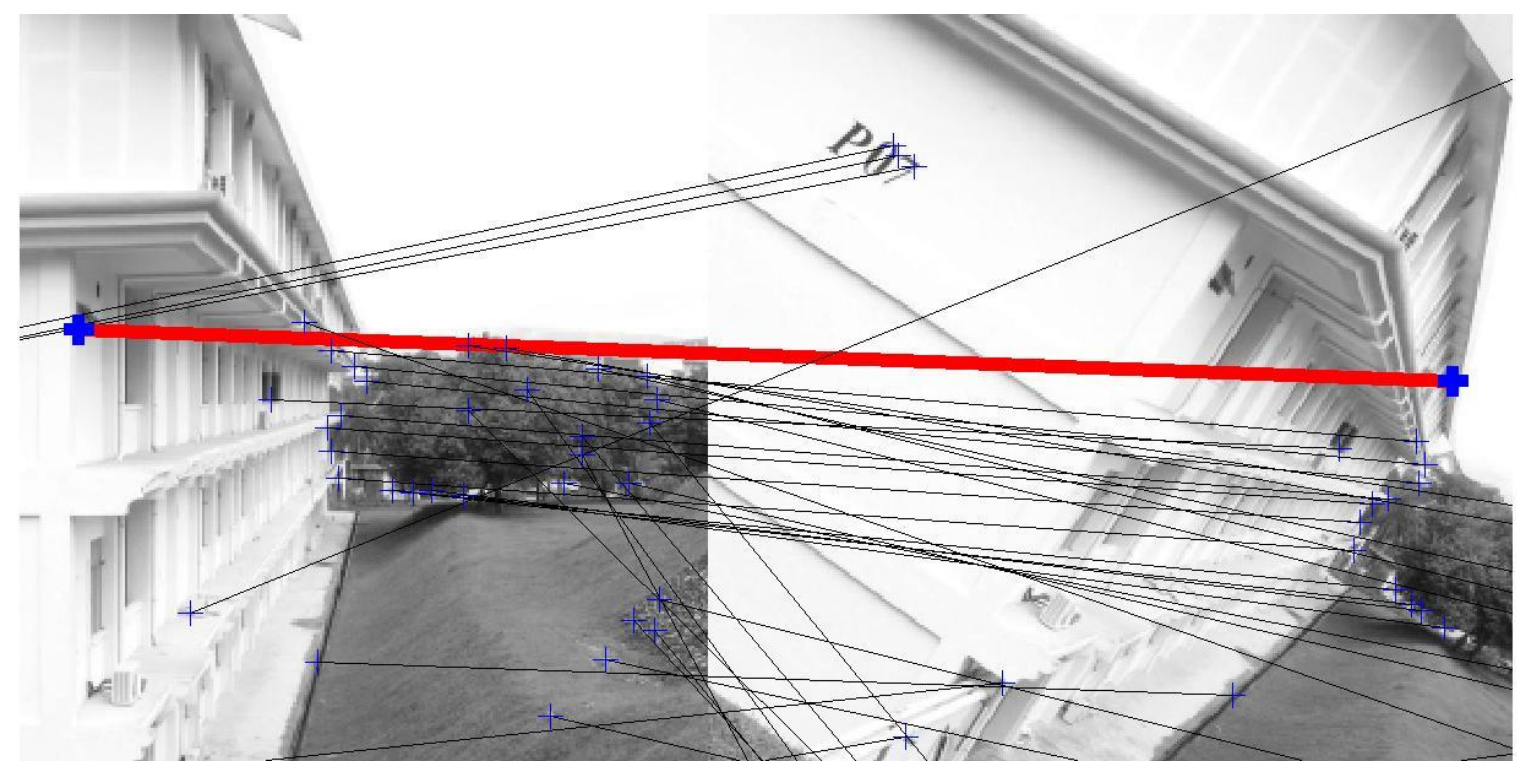

(a)

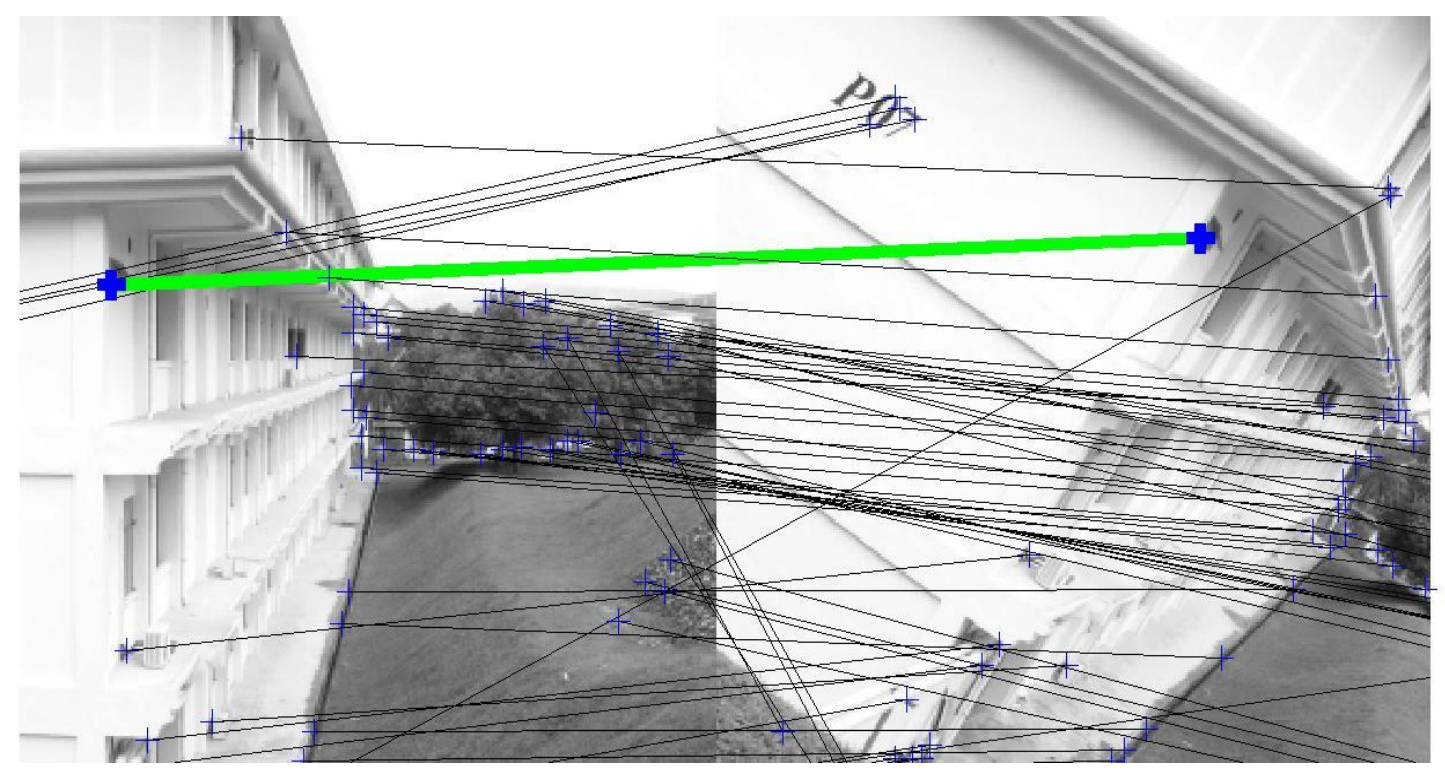

(b)

Fig. 6. (a) Before orientation correction, points on red line are falsely matched . (b) After orientation correction, matched points are corrected on green line.

This feedback process is iterated once only because, from our experiment, one loop is good enough to improve the number of correct matches. Comparison before and after the orientation correction is applied to the method is shown in Fig. 7. Fig. 7 (b) obviously has more matched points after applying the orientation correction as compared to Fig. 7(a). We note that the orientation difference histogram was also introduced in some previous works, such as in [28][29] where the orientation difference histogram was simply used to check the consistency of the matched pairs and improve 
their matching score, but global orientation was not used to increase the number of matched points. In this regard, our method is different from their work. We extend the idea by updating the descriptors, based on the global orientation generated from the histogram, to increase the matching rate for the number of correctly matched points at the same time.

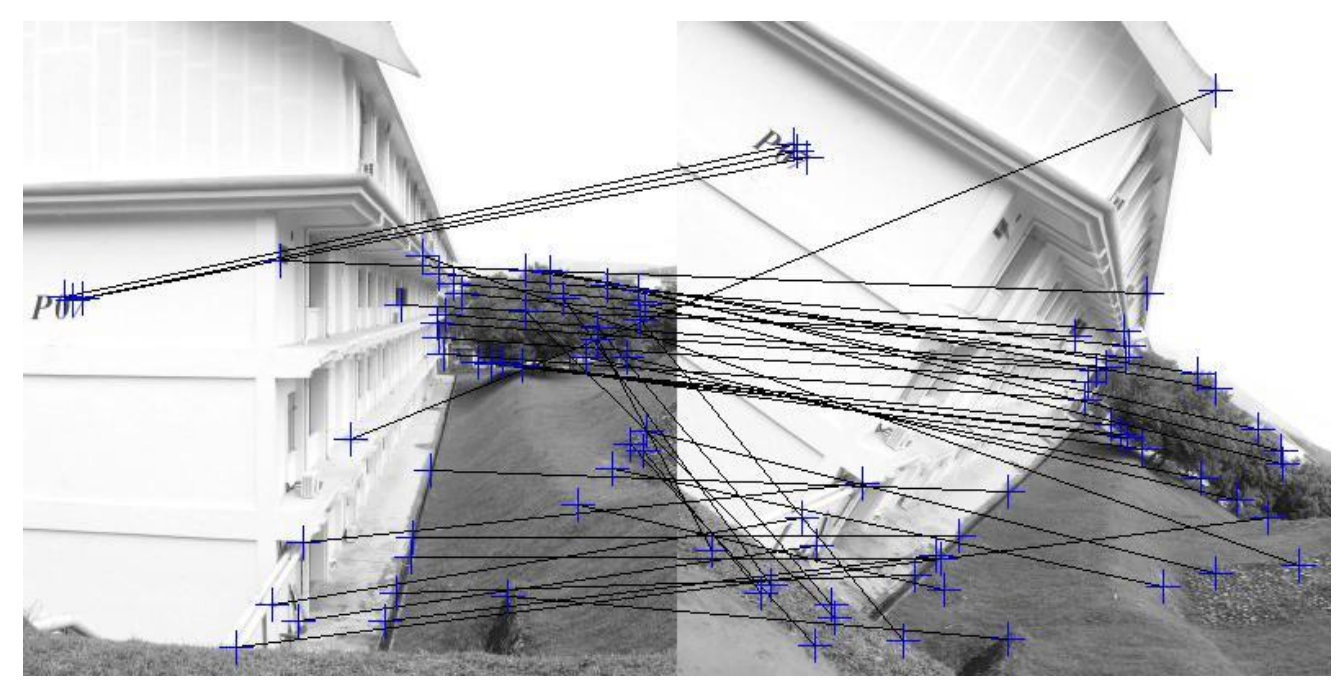

(a)

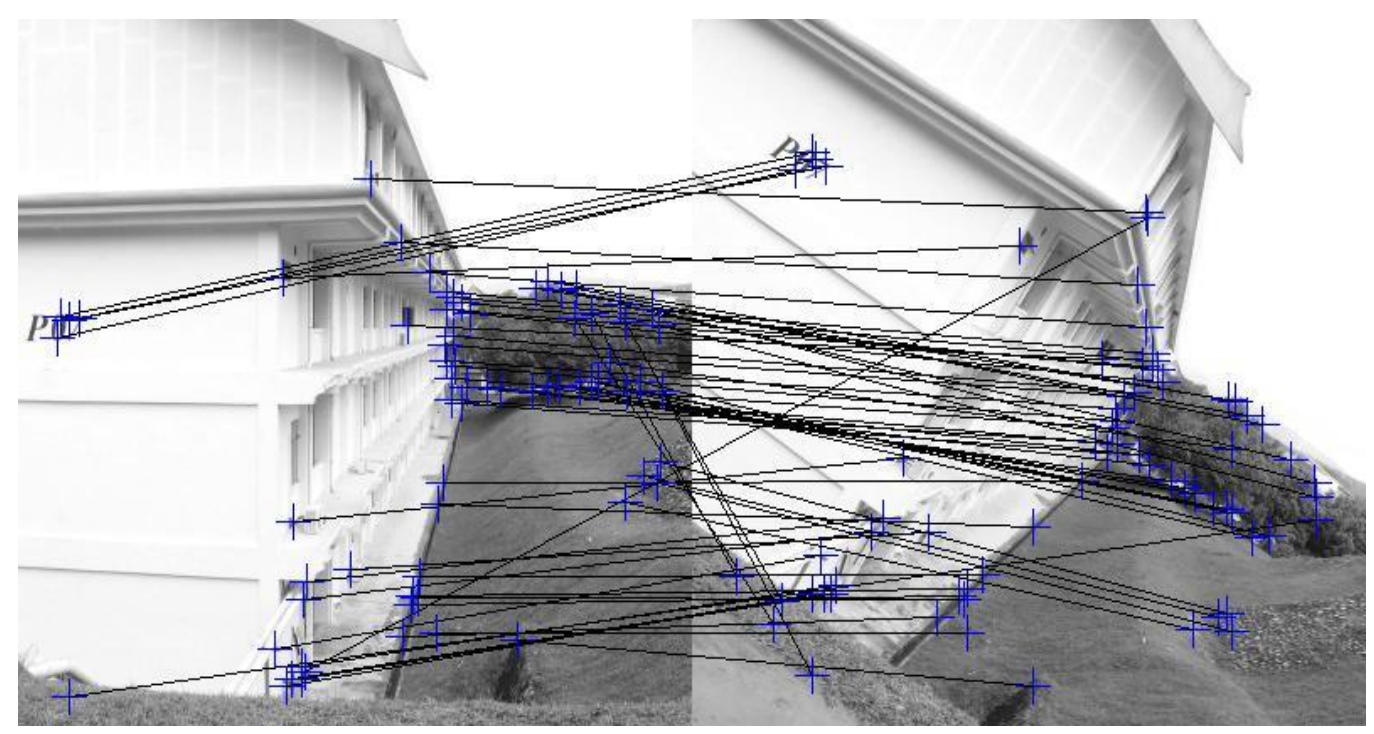

(b)

Fig. 7. Comparison of matched points (a) before (b) after orientation correction 


\section{Experimental Results and Discussion}

Our goal is to increase the true matched points while reducing the false matched points, as is needed to form the fundamental matrix or homography matrix in image warping. We therefore, evaluate the performance in two parts: the number of correct matches and the ratio between falsely matched points and correctly matched points before and after our proposed method is applied. For the second measurement, the higher the ratio value, the higher the number of false match points.

For performance comparison, we selected three sets of different outdoor scene images each one $800 \times 800$ pixels. In every set, samples with five different rotational angles of $30^{\circ}, 60^{\circ}, 90^{\circ}, 135^{\circ}$, and $180^{\circ}$ were taken to validate the robustness of the proposed technique. Therefore, the techniques for assigning orientation mentioned in Section 1 were used and all of them were adopted using the proposed method.

All of the images used in this experiment are taken from an outdoor scene. The first sample is an image of a simple scene which is then rotated in the five rotational angles mentioned above. The $90^{\circ}$ sample was rotated using image editing software so that the algorithm could be tested in a perfectly rotated scene without losing information. The other samples were taken by rotating the camera manually. The second sample was an image of a complex object close to the camera while the third sample was an image of a complex object taken from afar. With a complex image, false matching occurs more easily as there may be more features with similar descriptor values. We chose one simple scene sample image and two sample images with complex objects to verify that our proposed technique can work well with both simple scenes as well as with complex objects or backgrounds. We also considered both near-field and far-field images to test the effectiveness of our proposed method. In the case of the far-field view, the object of interest normally appears to be small. Hence, feature points tend to cluster very closely. Nevertheless, this would not be an obstacle for our method. Fig. 8 shows the image of the first sample and its rotated variants while Fig. 9 shows the sample of images with complex objects near to and far from the camera. One hundred and fifty points were detected from the first sample image and 164 points detected from the other two sample images. We also applied our proposed algorithm on the dataset given by Heinly in [30], in which the dataset contains eight images with different rotational degrees from $0^{\circ}$ to $180^{\circ}$. We chose six rotated images from the dataset and matched them with the original unrotated images to test the robustness of our proposed algorithm. Five hundred points were detected in the reference image of this dataset. Fig. 10 shows some of the samples from the dataset. In the following section, all correctly matched points are identified based on the human vision system.

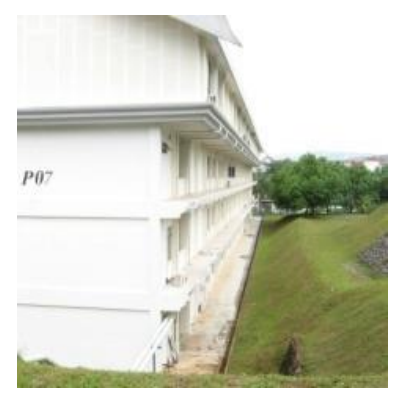

(a)

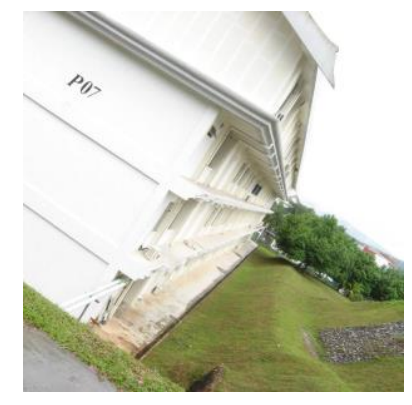

(b)

Fig. 8. A simple scene sample image at rotational angles of (a) $0^{\circ}$ and (b) $60^{\circ}$. 


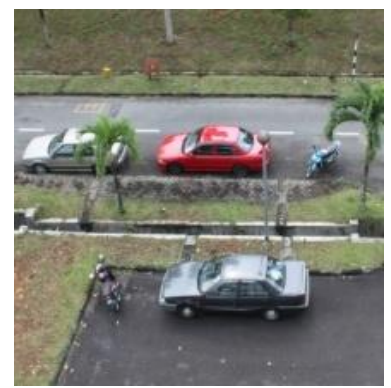

(a)

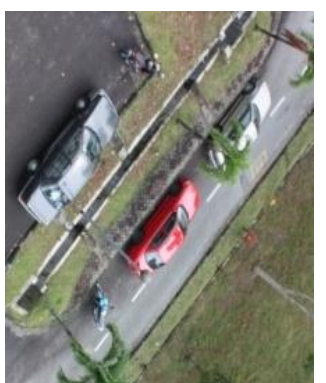

(b)

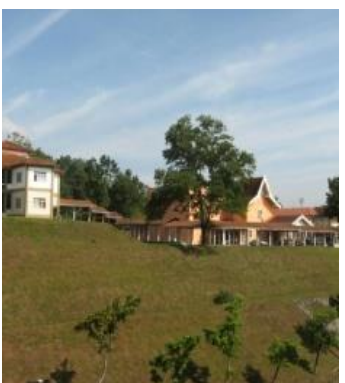

(c)

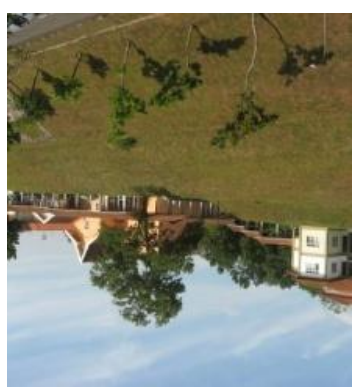

(d)

Fig.9. Complex scenes with $(a-b)$ near and $(c-d)$ far-field views.

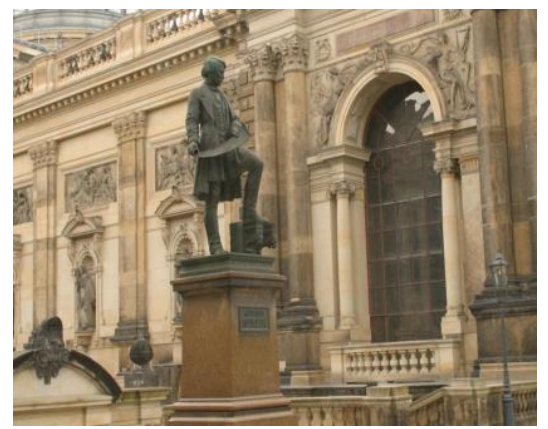

(a)

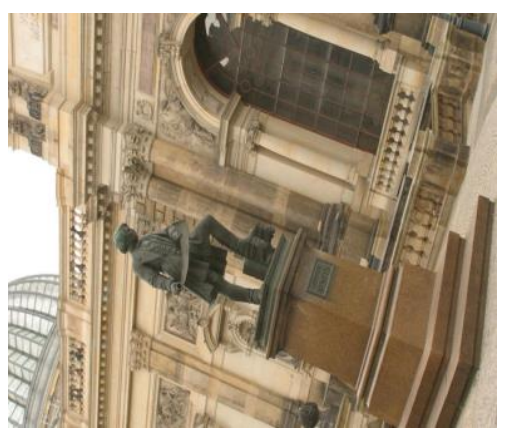

(b)

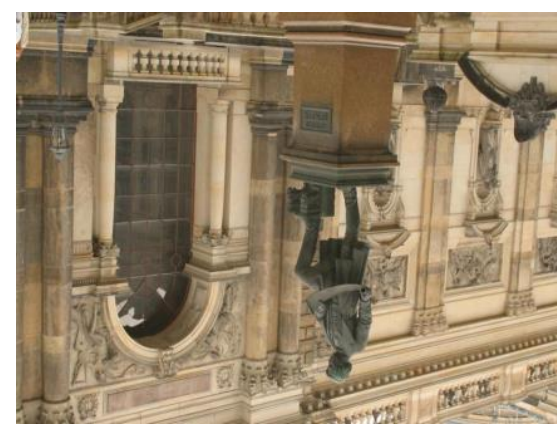

(c)

Fig.10. Rotated images from the Heinly dataset

\subsection{Results of a Simple Scene Image.}

Fig. 11 shows the comparison of the correctly matched points in all approaches.

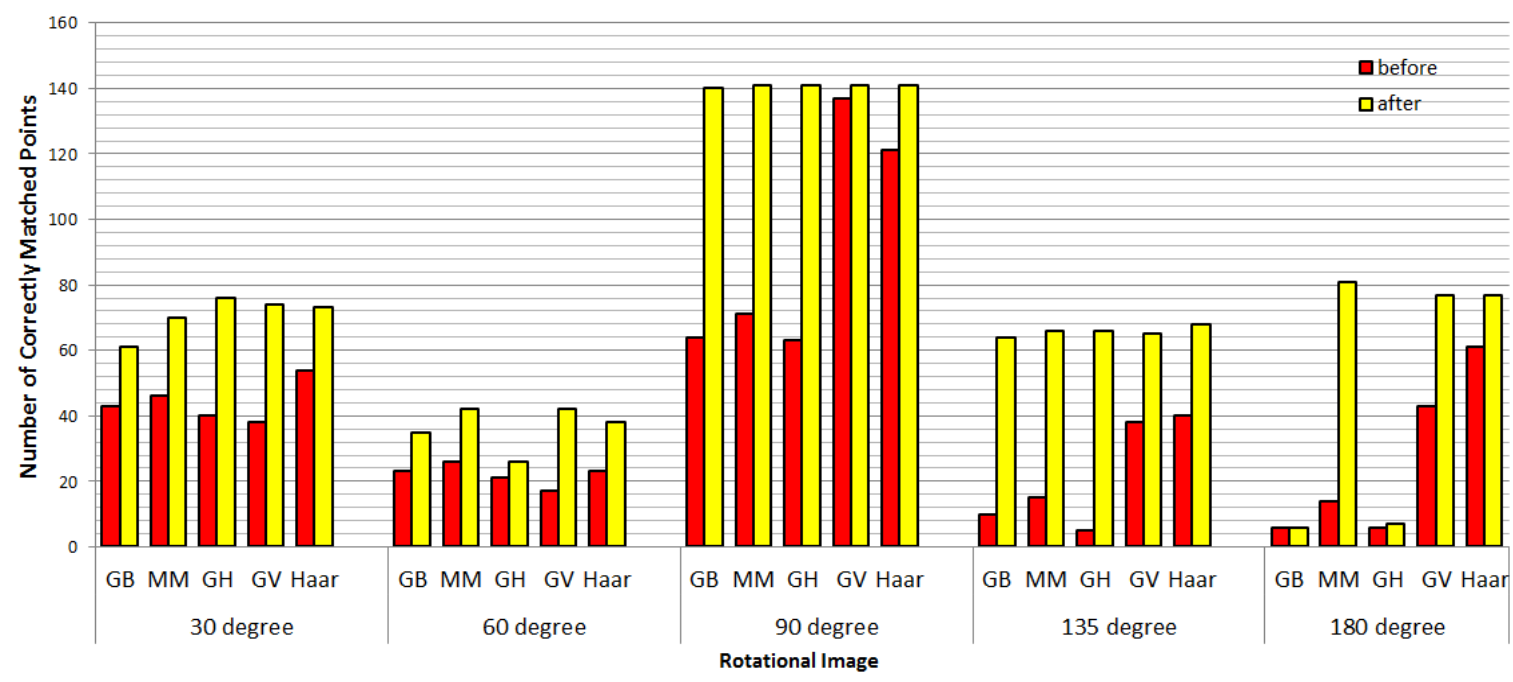

Fig.11. The comparison of the methods before and after applying orientation correction on a simple scene image 
From the above figure, we can see that Haar orientation assignment has the most correctly matched points compared to the other orientation assignment methods, except when the rotation is at $90^{\circ}$. At this orientation, gradient magnitude with the voting system and the Haar approaches both achieve a very high number of correctly matched points. This is because these two approaches are highly dependent on the vertical and horizontal information of the feature. In addition, since the $90^{\circ}$ rotation is achieved by computer, the image can be produced without noise. Therefore, the number of correctly matched points using these two approaches is among the highest. For other rotational angles, these two approaches perform equally well compared to the other three approaches which are weak in rotational matching, especially at $90^{\circ}, 135^{\circ}$ and $180^{\circ}$. However, by applying our orientation correction, the number of correctly matched points in all orientation assignment approaches has significantly increased. For rotation at $90^{\circ}$, our method is able to produce almost perfectly matched points which proves the effectiveness of our approach.

We also compared the ratio of falsely matched points over correctly matched points before and after orientation correction was applied, as shown in Fig. 12.

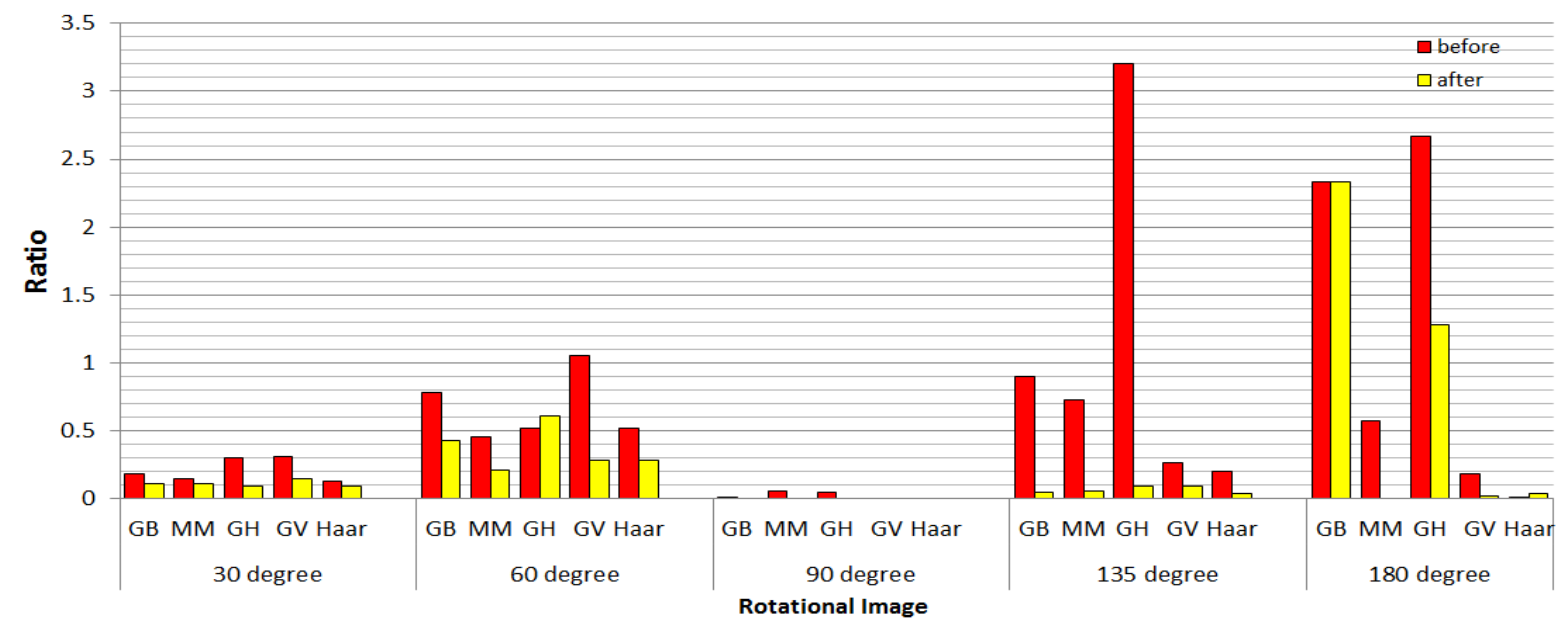

Fig. 12. Ratio of falsely matched points over correctly matched points before and after orientation correction is applied to a simple scene image sample

From Fig. 12, orientation correction is able to reduce the ratio of most of the samples especially for the $90^{\circ}$ rotational angle. The decrease in the ratio is due to the reduction in falsely matched points as well as the increment in correctly matched points. The falsely matched points in the $90^{\circ}$ sample are completely eliminated after applying our proposed method. For the $180^{\circ}$ sample, the ratios before and after GB orientation correction are the same. This is because the majority of matches in this sample are false matches. Table II and Table III show the numerical results of Fig. 11 and Fig. 12 respectively.

TABle II: NumericAl Result OF Number of MATChEd PoINTS IN SimPle SCENE IMAgE

\begin{tabular}{|l|l|l|l|l|l|l|l|l|l|l|}
\hline \multirow{2}{*}{ Approach } & \multicolumn{3}{|c|}{$30^{\circ}$} & \multicolumn{2}{c|}{$60^{\circ}$} & \multicolumn{3}{c|}{$90^{\circ}$} & \multicolumn{3}{c|}{$135^{\circ}$} & \multicolumn{2}{c|}{$180^{\circ}$} \\
\cline { 2 - 11 } & before & after & before & after & before & after & before & after & before & after \\
\hline GB & 43 & 61 & 23 & 35 & 64 & 140 & 10 & 64 & 6 & 6 \\
\hline MM & 46 & 70 & 26 & 42 & 71 & 141 & 15 & 66 & 14 & 81 \\
\hline GH & 40 & 76 & 21 & 26 & 63 & 141 & 5 & 66 & 6 & 7 \\
\hline GV & 38 & 74 & 17 & 42 & 137 & 141 & 38 & 65 & 43 & 77 \\
\hline Haar & 54 & 73 & 23 & 38 & 121 & 141 & 40 & 68 & 61 & 77 \\
\hline
\end{tabular}


TABLE III: NUMERICAL ReSUlt OF RATIO IN SIMPLE SCENE IMAGE

\begin{tabular}{|l|l|l|l|l|l|l|l|l|l|l|}
\hline \multirow{2}{*}{ Approach } & \multicolumn{2}{|c|}{$30^{\circ}$} & \multicolumn{2}{c|}{$60^{\circ}$} & \multicolumn{3}{c|}{$90^{\circ}$} & \multicolumn{2}{c|}{$135^{\circ}$} & \multicolumn{3}{c|}{$180^{\circ}$} \\
\cline { 2 - 11 } & before & after & before & after & before & after & before & after & before & after \\
\hline GB & 0.1860 & 0.1148 & 0.7826 & 0.4286 & 0.0156 & 0 & 0.9000 & 0.04688 & 2.3333 & 2.3333 \\
\hline MM & 0.1522 & 0.1143 & 0.4615 & 0.2143 & 0.0563 & 0 & 0.7333 & 0.06061 & 0.5714 & 0 \\
\hline GH & 0.3000 & 0.0921 & 0.5238 & 0.6154 & 0.0476 & 0 & 3.2000 & 0.09091 & 2.6667 & 1.2857 \\
\hline GV & 0.3158 & 0.1486 & 1.0588 & 0.2857 & 0 & 0 & 0.2632 & 0.09231 & 0.1860 & 0.0260 \\
\hline Haar & 0.1296 & 0.0959 & 0.5217 & 0.2895 & 0 & 0 & 0.2000 & 0.04412 & 0.0164 & 0.0390 \\
\hline
\end{tabular}

\subsection{Results for Complex Near-field Image.}

Once we had proven the effectiveness of our proposed method on a simple scene image, we extended the use of the method to a more challenging image. We began with a complex near-field image (Fig. 9(a)-(b)). Fig. 13 shows the experimental results for different orientation assignment approaches.

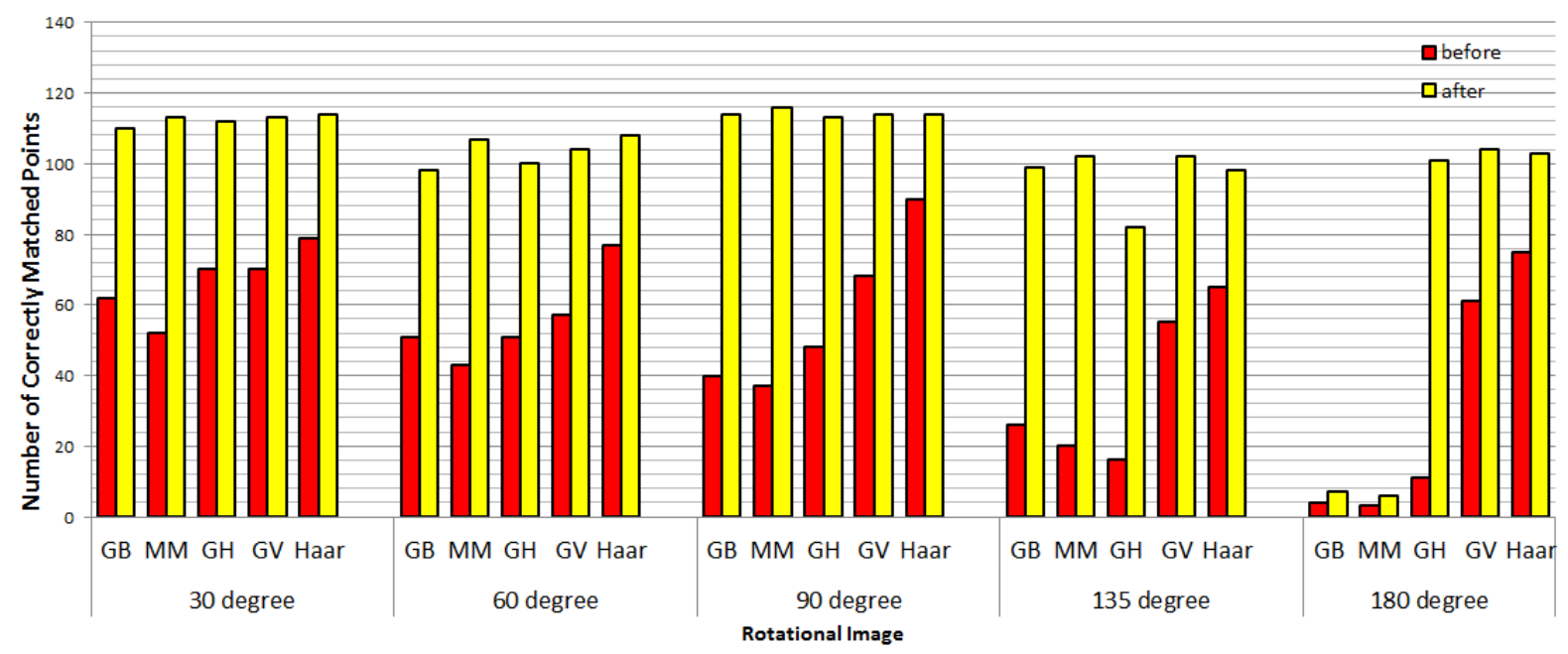

- $\quad$ Fig. 13. Comparison between before and after applying orientation correction on a complex nearfield image

- Fig.13 shows that the Haar orientation assignment method consistently produces more correct matches compared to other approaches while the gradient magnitude with the voting system gives the second highest results. Gabor and moment orientation assignment methods perform poorly at $180^{\circ}$ due to the few correctly matched pairs. The number of correctly matched points increased slightly in these two approaches using our orientation correction method. In addition, the number of correct matches for the first three approaches decreased when the rotational angle increased. The gradient magnitude with voting and Haar orientation assignments are not affected by the rotational angle. Fig. 14 shows the comparison of the ratios among all the approaches. 


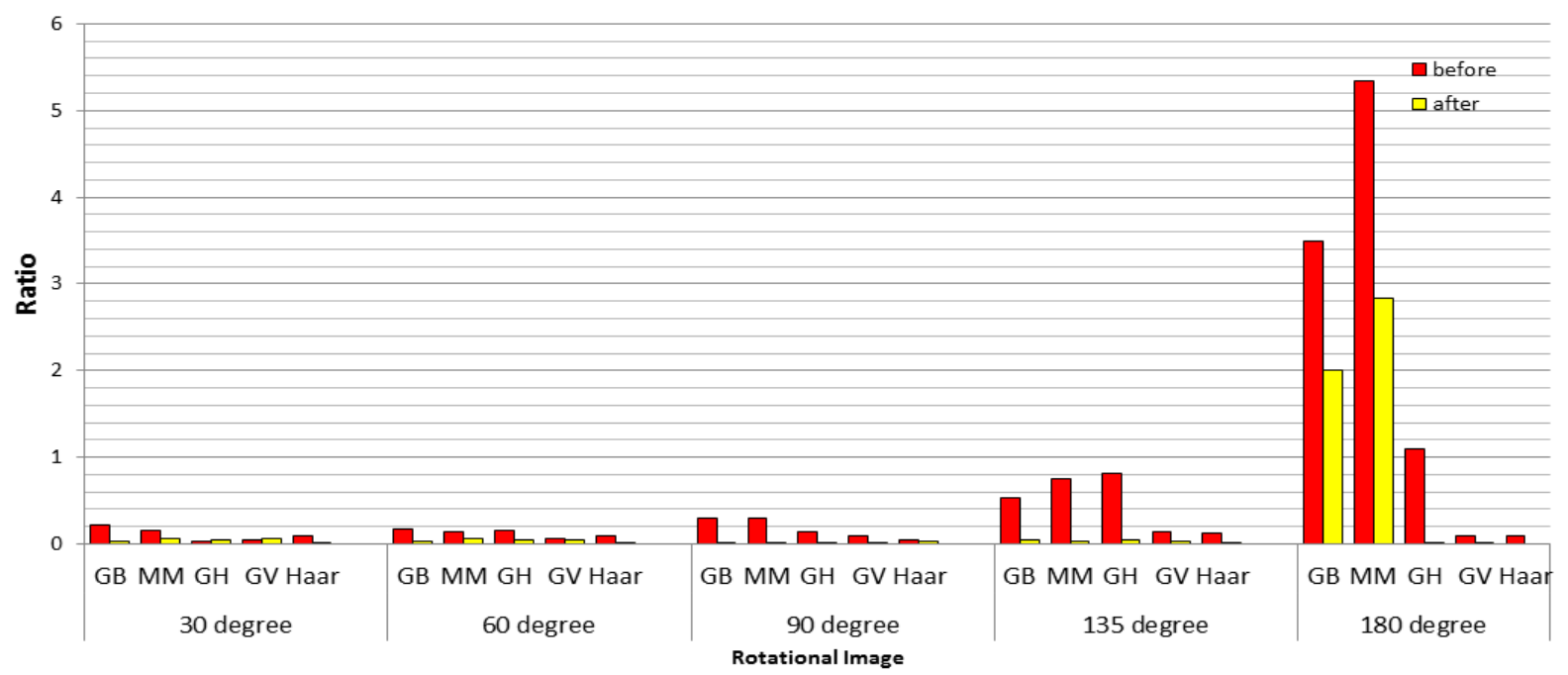

- $\quad$ Fig. 14. Comparison of the ratio of falsely matched points over correctly matched points before and after orientation correction for a complex near-field image.

The ratio of all approaches for the first four rotational angles of $30^{\circ}, 60^{\circ}, 90^{\circ}$ and $135^{\circ}$ degrees is almost zero after applying orientation correction. This shows that our proposed method is able to increase the number of correctly matched points, and at the same time reduce falsely matched pairs. At $180^{\circ}$, even though we may not be able to increase the number of correctly matched points for the Gabor and moment orientation assignment methods, our proposed method is still able to remove a certain number of false matches. This is a clear indication that our proposed method works well for complex images. We also summarized the numerical results of both comparisons and these are given in Table IV and Table $\mathrm{V}$ respectively.

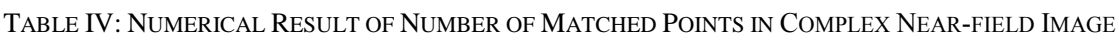

\begin{tabular}{|c|c|c|c|c|c|c|c|c|c|c|}
\hline \multirow[t]{2}{*}{ Approach } & \multicolumn{2}{|c|}{$30^{\circ}$} & \multicolumn{2}{|c|}{$60^{\circ}$} & \multicolumn{2}{|c|}{$90^{\circ}$} & \multicolumn{2}{|c|}{$135^{\circ}$} & \multicolumn{2}{|c|}{$180^{\circ}$} \\
\hline & before & after & before & after & before & after & before & after & before & after \\
\hline GB & 62 & 110 & 51 & 98 & 40 & 114 & 26 & 99 & 4 & 7 \\
\hline MM & 52 & 113 & 43 & 107 & 37 & 116 & 20 & 102 & 3 & 6 \\
\hline $\mathrm{GH}$ & 70 & 112 & 51 & 100 & 48 & 113 & 16 & 82 & 11 & 101 \\
\hline GV & 70 & 113 & 57 & 104 & 68 & 114 & 55 & 102 & 61 & 104 \\
\hline Haar & 79 & 114 & 77 & 108 & 90 & 114 & 65 & 98 & 75 & 103 \\
\hline
\end{tabular}


TABLE V: NuMERICAL RESUlt OF RATIO IN COMPLEX NEAR-FIELD IMAGE

\begin{tabular}{|l|l|l|l|l|l|l|l|l|l|l|l|}
\hline \multirow{2}{*}{ Approach } & \multicolumn{2}{|c|}{$30^{\circ}$} & \multicolumn{2}{c|}{$60^{\circ}$} & \multicolumn{2}{c|}{$90^{\circ}$} & \multicolumn{2}{c|}{$135^{\circ}$} & \multicolumn{2}{c|}{$180^{\circ}$} \\
\cline { 2 - 11 } & before & after & before & after & before & after & before & after & before & after \\
\hline GB & $\begin{array}{l}0.2 \\
26\end{array}$ & 0.027 & 0.177 & 0.031 & 0.3 & 0.009 & 0.536 & 0.040 & 3.5 & 2 \\
\hline MM & $\begin{array}{l}0.1 \\
54\end{array}$ & 0.071 & 0.140 & 0.056 & 0.297 & 0.009 & 0.750 & 0.039 & 5.333 & 2.833 \\
\hline GH & $\begin{array}{l}0.0 \\
29\end{array}$ & 0.045 & 0.160 & 0.04 & 0.146 & 0.018 & 0.813 & 0.049 & 1.091 & 0.010 \\
\hline GV & $\begin{array}{l}0.0 \\
43\end{array}$ & 0.062 & 0.07 & 0.048 & 0.088 & 0.018 & 0.146 & 0.039 & 0.098 & 0.010 \\
\hline Haar & 0.1 & 0.009 & 0.091 & 0.019 & 0.044 & 0.026 & 0.124 & 0.020 & 0.093 & 0 \\
\hline
\end{tabular}

\subsection{Results for Complex Far-field Image.}

Our final test was to apply the proposed method to even more challenging images, i.e. complex farfield images. Fig. 15 shows the results of our test.

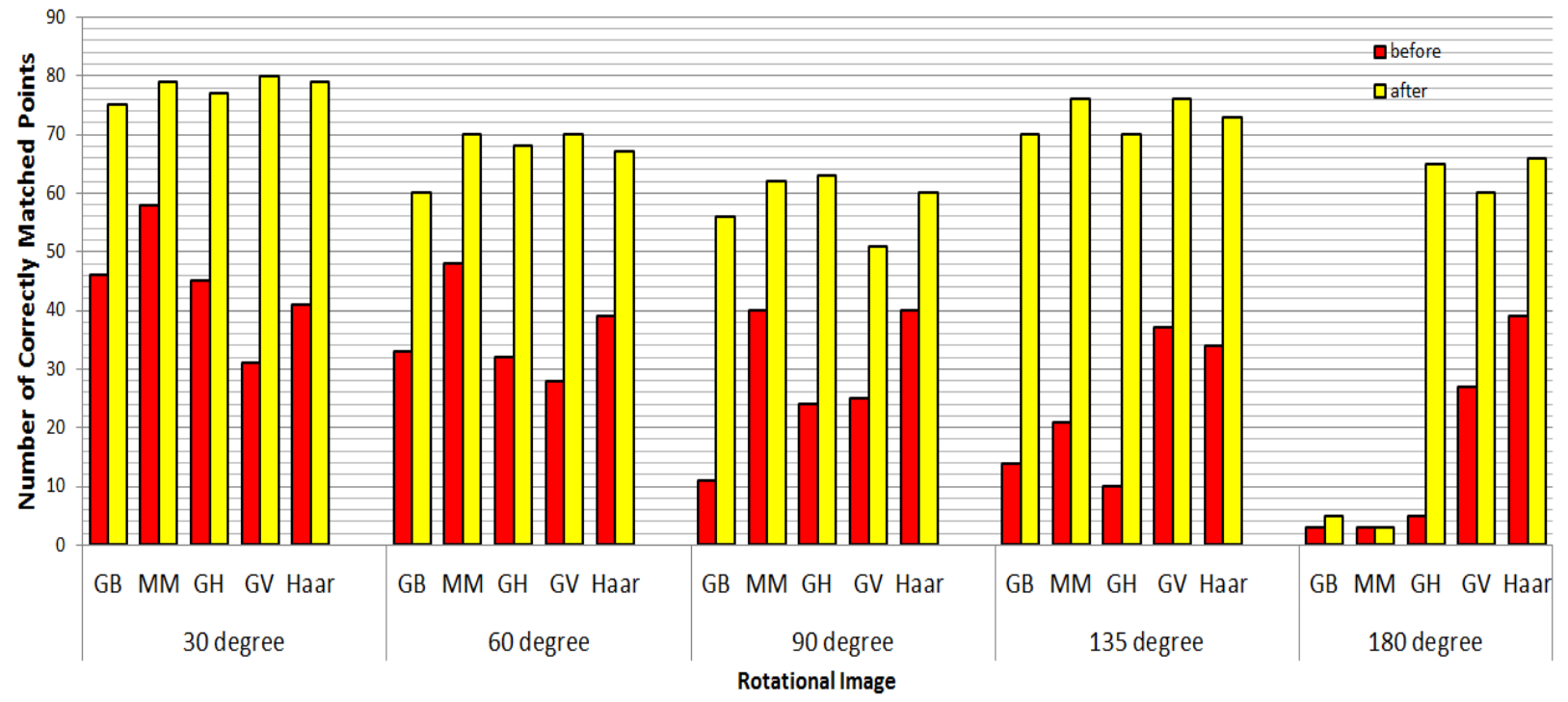

Fig. 15. Comparison among different orientation assignment methods before and after applying orientation correction to a complex far-field image.

Unlike in the previous tests, the first three rotational angles of $30^{\circ}, 60^{\circ}$ and $90^{\circ}$ produced more correctly matched points for the Gabor and moment orientation assignment methods than with gradient magnitude with voting and Haar. However, the Gabor, moment and gradient magnitude with hierarchy implementation approaches still suffer from a decrease in the number of correctly matched points when the rotational angle is increased. In terms of stability, Haar and gradient magnitude with voting are better than the others. With the incorporation of our proposed method, the numbers of correctly matched points has significantly increased. Meanwhile, Fig. 16 shows the ratio between the false and correctly matched pairs. 


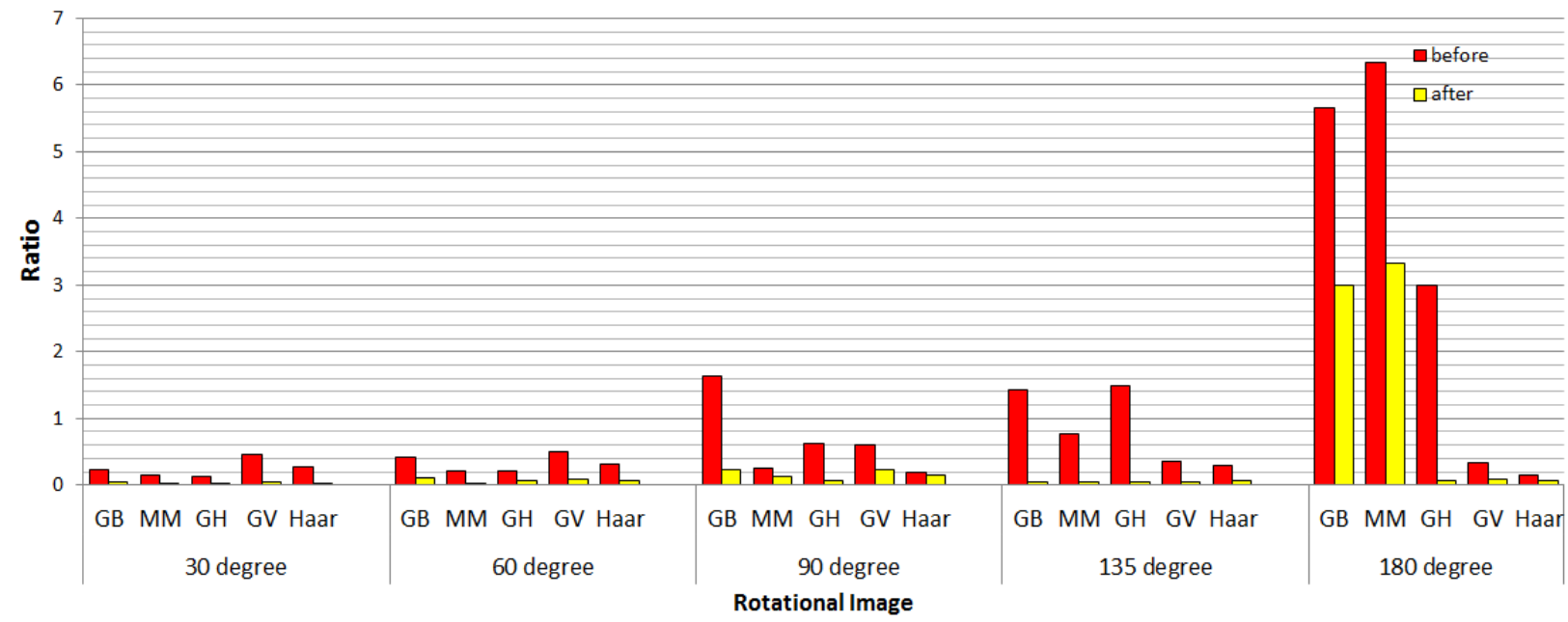

Fig. 16. Ratio of falsely matched points over correctly matched points before and after orientation correction of complex far-field image.

In Fig. 16, our proposed method managed to reduce the number of falsely matched pairs while increasing the number of correctly matched pairs. The ratios for all rotational angles, except for the rotational angle of $180^{\circ}$, decreased to almost zero after applying orientation correction. As with the two previous sections, we show the results in table form for easy visualization in Table VI and Table VII.

TABLE VI: NumericAl Result of Number of MATCHEd PoINTS IN COMPLEX FAR-FIELD IMAGE

\begin{tabular}{|l|l|l|l|l|l|l|l|l|l|l|}
\hline \multirow{2}{*}{ Approach } & \multicolumn{3}{|c|}{$30^{\circ}$} & \multicolumn{2}{c|}{$60^{\circ}$} & \multicolumn{2}{c|}{$90^{\circ}$} & \multicolumn{2}{c|}{$135^{\circ}$} & \multicolumn{2}{c|}{$180^{\circ}$} \\
\cline { 2 - 11 } & before & after & before & after & before & after & before & after & before & after \\
\hline GB & 46 & 75 & 33 & 60 & 11 & 56 & 14 & 70 & 3 & 5 \\
\hline MM & 58 & 79 & 48 & 70 & 40 & 62 & 21 & 76 & 3 & 3 \\
\hline GH & 45 & 77 & 32 & 68 & 24 & 63 & 10 & 70 & 5 & 65 \\
\hline GV & 31 & 80 & 28 & 70 & 25 & 51 & 37 & 76 & 27 & 60 \\
\hline Haar & 41 & 79 & 39 & 67 & 40 & 60 & 34 & 73 & 39 & 66 \\
\hline
\end{tabular}

TABLE VII: NUMERICAL RESULT OF RATIO IN COMPLEX FAR-FIELD IMAGE

\begin{tabular}{|l|l|l|l|l|l|l|l|l|l|l|}
\hline \multirow{2}{*}{ Approach } & \multicolumn{2}{|c|}{$30^{\circ}$} & \multicolumn{3}{c|}{$60^{\circ}$} & \multicolumn{3}{c|}{$90^{\circ}$} & \multicolumn{3}{c|}{$135^{\circ}$} & \multicolumn{2}{c|}{$180^{\circ}$} \\
\cline { 2 - 10 } & before & after & before & after & before & after & before & after & before & after \\
\hline GB & 0.239 & 0.04 & 0.424 & 0.117 & 1.636 & 0.232 & 1.428 & 0.057 & 5.667 & 3 \\
\hline MM & 0.155 & 0.025 & 0.208 & 0.029 & 0.25 & 0.129 & 0.762 & 0.053 & 6.333 & 3.333 \\
\hline GH & 0.133 & 0.026 & 0.218 & 0.059 & 0.625 & 0.063 & 1.5 & 0.057 & 3 \\
\hline GV & 0.452 & 0.038 & 0.5 & 0.086 & 0.6 & 0.235 & 0.351 & 0.0523 & 0.333 & 0.083 \\
\hline Haar & 0.268 & 0.025 & 0.308 & 0.060 & 0.2 & 0.15 & 0.294 & 0.068 & 0.154 & 0.061 \\
\hline
\end{tabular}

\subsection{Results on Semper Dataset.}

In order to confirm our achievement, we also tested our proposed algorithm with the Semper public dataset [30]. Fig. 17 shows the comparison of correctly matched points before and after applying our algorithm. Since the dataset does not provide any information on the rotational angle of each 
image, the $\mathrm{x}$-axis of the histogram in Fig. 17 is arranged from approximately $10^{\circ}$ (img2) to $180^{\circ}$ (img9).

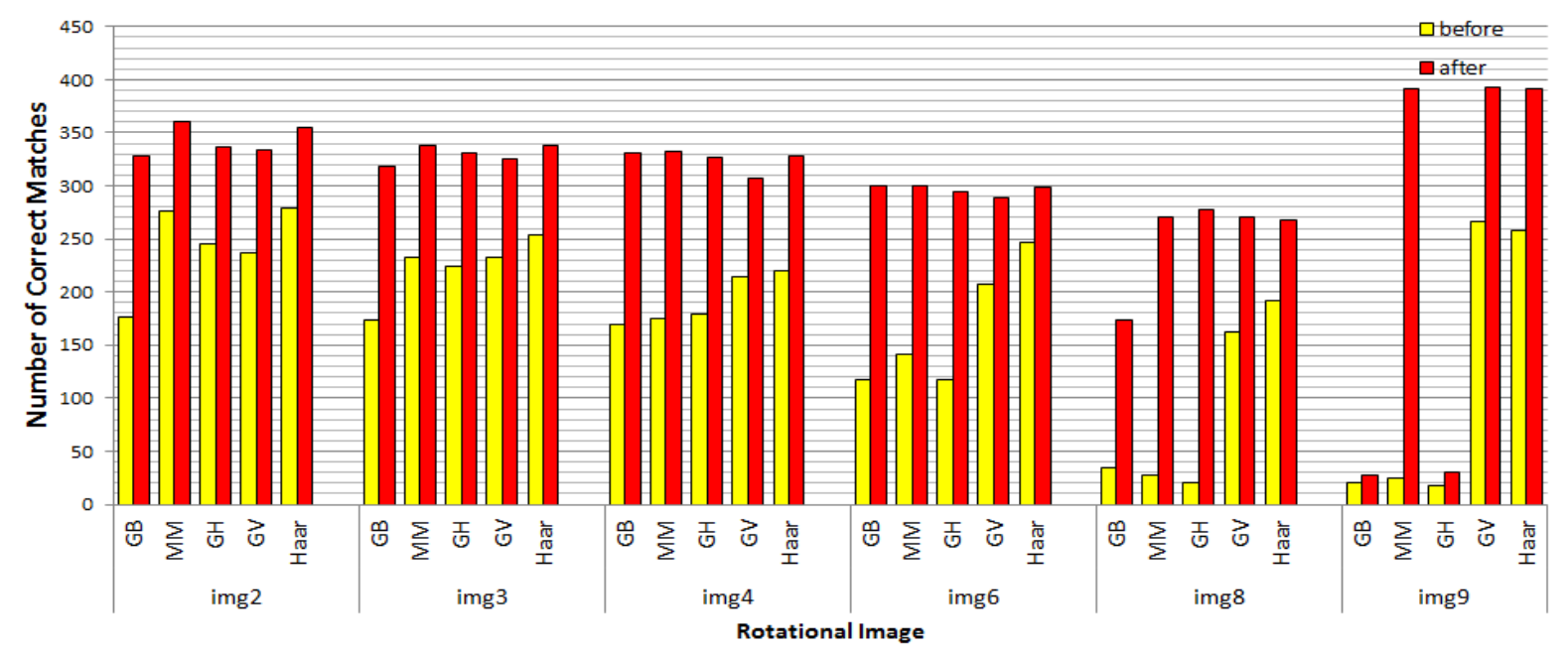

Fig. 17. Comparison among different orientation assignment methods before and after applying orientation correction to a public dataset.

From these experiments, we can generalize that, except for sample img9, the numbers of correctly matched points increased with our proposed method. As mentioned previously, the performance of the algorithm will result in a low number of correctly matched points if the initial matchings are mostly incorrect. Therefore, the results of the Gabor filter and the gradient with hierarchy implementation in img9 $\left(180^{\circ}\right)$ are not satisfactory compared to the other samples. Unlike previous experiments, the results from img2 to img8 are quite consistent after applying orientation correction. In addition, the ratios before and after orientation correction are also compared and shown in Fig. 18.

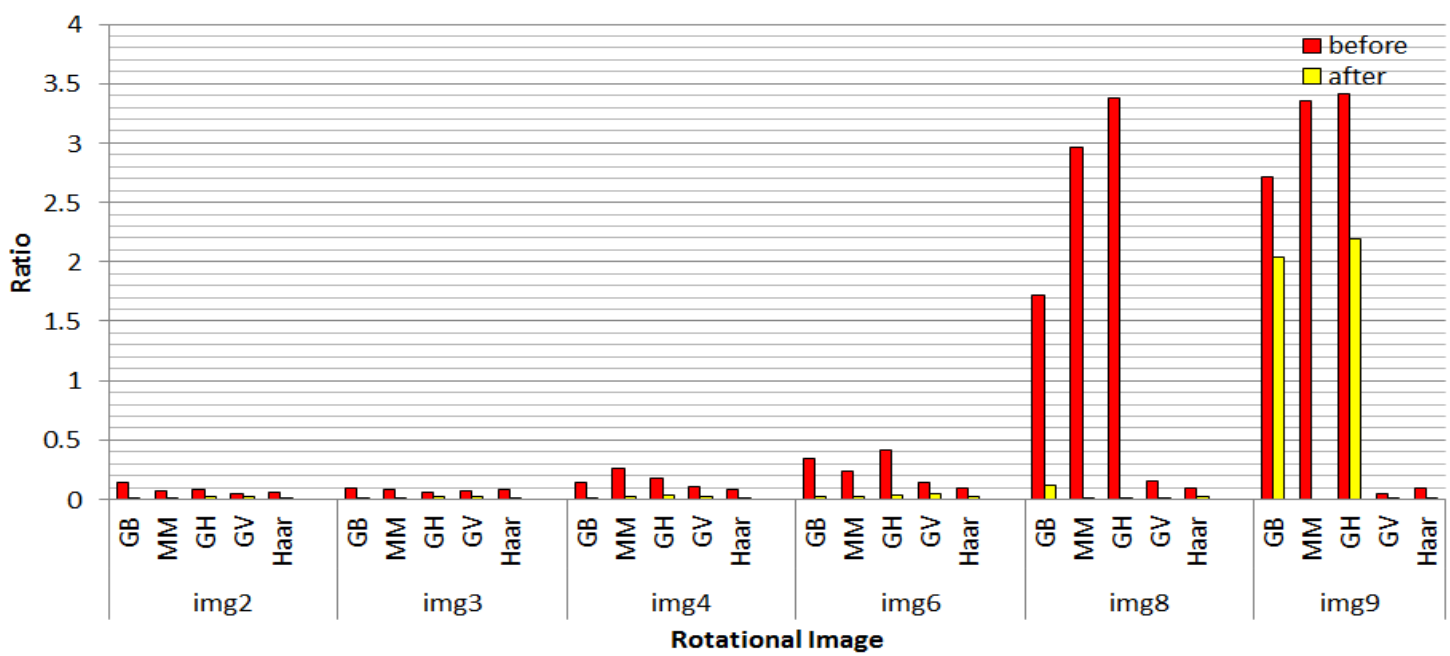

Fig. 18. Ratio of falsely matched points over correctly matched points before and after orientation correction on public dataset

From this figure, except img9, the ratios are close to zero after applying orientation correction to each sample. This shows that our method successfully removes incorrectly matched points and at the same time increases the number of correctly matched points even if the correctly matched points 
are fewer in the initial matching, as long as the majority of matched points are correct. Table VIII and Table IX illustrate the numerical comparison.

TABLE VIII: NuMERICAL ReSUlt OF NuMBER OF MATCHEd POINTS IN COMPLEX FAR-FIELd IMAGE

\begin{tabular}{|c|c|c|c|c|c|c|c|c|c|c|c|c|}
\hline \multirow{2}{*}{ Approach } & \multicolumn{2}{|c|}{ Img2 } & \multicolumn{2}{|c|}{ Img3 } & \multicolumn{2}{|c|}{ Img4 } & \multicolumn{2}{|c|}{ Img6 } & \multicolumn{2}{|c|}{ Img8 } & \multicolumn{2}{|c|}{ Img9 } \\
\hline & before & after & before & after & before & after & before & after & before & after & before & after \\
\hline GB & 176 & 328 & 173 & 319 & 169 & 331 & 118 & 300 & 35 & 174 & 21 & 28 \\
\hline MM & 276 & 360 & 232 & 338 & 175 & 332 & 141 & 300 & 28 & 271 & 25 & 392 \\
\hline $\mathrm{GH}$ & 245 & 336 & 224 & 331 & 179 & 327 & 117 & 295 & 21 & 278 & 17 & 31 \\
\hline GV & 237 & 334 & 233 & 325 & 214 & 307 & 207 & 289 & 162 & 271 & 267 & 393 \\
\hline Haar & 279 & 355 & 254 & 338 & 220 & 328 & 247 & 299 & 192 & 268 & 258 & 391 \\
\hline
\end{tabular}

TABLE IX: NumERICAL RESUlt OF RATIO IN COMPLEX FAR-FIELD IMAGE

\begin{tabular}{|c|c|c|c|c|c|c|c|c|c|c|c|c|}
\hline \multirow{2}{*}{$\begin{array}{l}\text { Approac } \\
\mathrm{h}\end{array}$} & \multicolumn{2}{|c|}{ Img2 } & \multicolumn{2}{|c|}{ Img3 } & \multicolumn{2}{|c|}{$\operatorname{Img} 4$} & \multicolumn{2}{|c|}{ Img6 } & \multicolumn{2}{|c|}{ Img8 } & \multicolumn{2}{|c|}{ Img9 } \\
\hline & $\begin{array}{l}\text { befor } \\
\text { e }\end{array}$ & after & $\begin{array}{l}\text { befor } \\
\text { e }\end{array}$ & after & $\begin{array}{l}\text { befor } \\
\text { e }\end{array}$ & after & $\begin{array}{l}\text { befor } \\
\text { e }\end{array}$ & after & $\begin{array}{l}\text { befor } \\
\text { e }\end{array}$ & after & $\begin{array}{l}\text { befor } \\
\text { e }\end{array}$ & after \\
\hline GB & 0.148 & $\begin{array}{l}0.01 \\
5\end{array}$ & 0.093 & $\begin{array}{l}0.00 \\
6\end{array}$ & 0.142 & $\begin{array}{l}0.01 \\
5\end{array}$ & 0.339 & $\begin{array}{l}0.02 \\
3\end{array}$ & 1.714 & $\begin{array}{l}0.12 \\
1\end{array}$ & 2.714 & $\begin{array}{l}2.035 \\
7\end{array}$ \\
\hline MM & 0.069 & $\begin{array}{l}0.00 \\
3\end{array}$ & 0.086 & $\begin{array}{l}0.01 \\
8\end{array}$ & 0.263 & $\begin{array}{l}0.02 \\
7\end{array}$ & 0.232 & 0.02 & 2.964 & $\begin{array}{l}0.01 \\
4\end{array}$ & 3.36 & 0 \\
\hline GH & 0.082 & $\begin{array}{l}0.02 \\
1\end{array}$ & 0.058 & $\begin{array}{l}0.02 \\
4\end{array}$ & 0.173 & $\begin{array}{l}0.03 \\
1\end{array}$ & 0.419 & $\begin{array}{l}0.03 \\
4\end{array}$ & 3.381 & $\begin{array}{l}0.01 \\
8\end{array}$ & 3.412 & 2.194 \\
\hline GV & 0.051 & $\begin{array}{l}0.02 \\
1\end{array}$ & 0.073 & $\begin{array}{l}0.02 \\
8\end{array}$ & 0.103 & $\begin{array}{l}0.02 \\
9\end{array}$ & 0.140 & $\begin{array}{l}0.04 \\
5\end{array}$ & 0.154 & $\begin{array}{l}0.00 \\
7\end{array}$ & 0.052 & 0.003 \\
\hline Haar & 0.057 & $\begin{array}{l}0.00 \\
8\end{array}$ & 0.079 & $\begin{array}{l}0.01 \\
2\end{array}$ & 0.086 & $\begin{array}{l}0.00 \\
9\end{array}$ & 0.093 & $\begin{array}{l}0.02 \\
7\end{array}$ & 0.094 & $\begin{array}{l}0.01 \\
9\end{array}$ & 0.093 & 0.010 \\
\hline
\end{tabular}

In the simple scene image, the objects were mostly in uniform regions such as the label "P07" on the building wall. As a result, less feature points were extracted since we used Harris Corner detector to extract corner points. Therefore, the number of total matched points from the first matching was relatively fewer compared to the other two complex images. The improvements after applying orientation correction were not as good as the other two complex image samples. In the complex images, several objects looked similar, such as vehicles and trees. More feature points were extracted and this resulted in more totally matched points. The number of correctly matched points of the matching in the complex near-field images was generally higher. This was because the objects sizes in the near-field images were greater than the objects sizes of the far-field objects. Edges and corners of the near-field images were easier to detect. In Fig. 13 (near-field samples), the correctly matched points increased from 82 to 116 points after orientation correction had been applied. In Fig. 15 (far-field samples), the number of correctly matched points increased from 51 to 80. For the Semper dataset, the increment of correctly matched points was between 84 and 370 . For the simple field sample, the increment percentage was around $10 \%$ to $50 \%$. In the other two samples, the increment percentage was in the range of $12 \%$ to $36 \%$ for the near-field samples and $14 \%$ to $54 \%$ for far-view samples. For the Semper dataset, the increment percentage was in the 
range of $20 \%$ to $50 \%$. Even though the results in the simple, near field and far field images were different, our proposed method managed to improve the numbers of correctly matched points. However, the orientation correction algorithm is unable to increase the number of correctly matched points if the majority of matched pairs is incorrect, since the proposed method is based on a single relative rotational angle. The increment in the number of matched points and the percentages are summarized in Table III.

\section{Conclusion}

This paper proposed a new method for correcting feature orientation so that the number of correctly matched points increases. We introduced a method that can find corresponding matched pairs by computing the relative rotational angle from the orientation difference histogram and regenerating new descriptors for matching based on the computed relative angle. Our method can increase the number of correctly matched points by up to $50 \%$ of the detected points in the reference image. It works well with simple object images and complex object images. In addition, the proposed methods managed to correct falsely matched to correctly matched images. Nevertheless, there are two issues that need to be addressed. First, the complexity of the system is yet to be determined. Second, if the majority of points is not matched correctly, the number of correctly matched points after applying the proposed method will be degraded. In our future work we plan to compare our work with some popular methods for removing outliers, such as the Random Sample Consensus (RANSAC) method. In addition, instead of the single relative rotational angle, orientation correction based on multiple relative rotational angles may be the future path to increasing matching robustness. For details of the ground truth specification, we noticed that RANSAC can be used to remove all outliers so that the remaining matched points will be the ground truth of matching as in [31]. The details of ground truth for every dataset will be provided by email by the author of this paper. For the inquiry of ground truth for every dataset, please kindly email us for further details.

\section{Acknowledgement}

This work was supported by the Universiti Teknologi Malaysia (UTM) and the Ministry of Higher Education (MoHE) under University Research Grant Q.J13000.7123.00H53

\section{References}

[1] S. J. Hu, Y. L Hu, Z. H. Chen, P. Jiang, "Feature-based image automatic mosaicing algorithm," The $6^{\text {th }}$ World Congress on Intelligent Control and Automation 2006, pp. 10361-10364.

[2] C. M Wen, T. D. Guo, S. G. Wang, "Fingerprint feature-point matching based on motion coherence," $2^{\text {nd }}$ Int. Conf. on Future Information Technology and Management Engineering 2009, pp. 226-229.

[3] Y. L. Guo, S. Hsu, H. S. Sawhney, R. Kumar, Y. Shan, "Robust object matching for persistent tracking with heterogeneous features," IEEE Transactions on Pattern Analysis and Machine Intelligence, vol. 29(5), pp. 824-839, 2007.

[4] V. Sulic, J. Pers, M. Kristan, S. Kovacic, "Efficient feature distribution for object matching in visual-sensor networks," IEEE Transactions on Circuits and Systems for Video Technology, vol. 21(7), pp. 903-916, July 2011.

[5] C. Khmanee, D. Nguyen, "On the design of 2D Gabor filtering of fingerprint images," $1^{s t}$ IEEE Consumer Communications and Networking Conf., 2004, pp. 430-435.

[6] D. G. Lowe, "Distinctive image features from scale-invariant key points," International Journal of Computer Vision, vol. 60, pp. 91-110, 2004.

[7] Z. H Huang, J. S. Leng, "Analysis of Hu's moment invariants on image scaling and rotation," $2^{\text {nd }}$ Int. Conf. on Computer Engineering and Technology, 2010, pp. V7-476-V7-480. 
[8] A. R. Rao, A Taxonomy for Texture Description and Identification, New York: SpringerVerlag, 1990.

[9] H. Bay, A. Ess, T. Tuytelaars, L. V. Gool, "SURF: speeded up robust features," Computer Vision and Image Understanding, vol. 110(3), pp. 346-359, 2008.

[10] G. Zhang, Z. M. Ma, "Texture feature extraction and description using Gabor wavelet in content-based medical image retrieval," Int. Conf. on Wavelet Analysis and Pattern Recognition, 2007, pp. $169-173$.

[11] J. K. Kamarainen, V. Kyrki, H. Kalviainen, "Invariance properties of Gabor filter-based features-overview and applications," IEEE Transactions on Image Processing, vol. 1(5), pp. 10881099, 2005.

[12] B. Popovic, M. Bandjur, A. Raicevic, "Robust enhancement of fingerprint images obtained by ink method," Electronics Letters, vol. 46(20), pp. 1379-1380, 2010.

[13] J. M. C. Sousa, J. M. Gil, J. R. C. Pinto. "Word indexing of ancient documents using fuzzy classification," IEEE Transactions on Fuzzy Systems, vol. 15, pp. 852-862, 2007.

[14] F. Oloumi, R. M. Rangayyan, A. L. Ells, "Parabolic modeling of the major temporal arcade in retinal fundus images," IEEE Transactions on Instrumentation and Measurement, vol. 61, pp. 1825-1838, 2012.

[15] V. Asha, N. U. Bhajantri, P. Nagabhushan, "Automatic detection of texture defects using texture-periodicity and Gabor wavelets," Computer Networks and Intelligent Computing, vol. 157, pp. $548-553,2011$.

[16] H. Ming-Kuei, "Visual pattern recognition by moment invariants," IRE Transactions Information Theory, vol. 8, pp. 179-187, 1962.

[17] A. Begum, D. Manimegalai, A. Abudhahir, "Optimum coefficients of discrete orthogonal tchebichef moment transform to improve the performance of image compression," Malaysian Journal of Computer Science, vol. 26(1), pp. 60-75, 2013.

[18] N. Kumar, A. C. Lammert, B. Englot, F. S. Hover, S. S. Narayanan, "Directional descriptors using zernike moment phases for object orientation estimation in underwater sonar images," IEEE Int. Conf. on Acoustics, Speech and Signal Processing, pp. 1025-1028, May 2011.

[19] M. Sassano, D. Carnevale, A. Astolfi, "A geometric moment-based observer for global range and local orientation identification," American Control Conference, 2010, pp. 178-183.

[20] Y. S. Abu-Mostafa, D. Psaltis, "Recognitive aspects of moment invariants," IEEE Transactions on Pattern Analysis and Machine Intelligence, vol. 6, pp. 698-706, 1984.

[21] M. Sadeghi, T. K. Lee, "Oriented pattern analysis for streak detection in dermoscopy images," Proc. of the $15^{\text {th }}$ Int. Conf. on Medical Image Computing and Computer-Assisted Intervention, 2012, Part I pp. 298-306.

[22] J. W.Yang, L. F. Liu, T. Z. Jiang, "Improved method for extraction of fingerprint features," Proc. of $2^{N D}$ Int. Conf. on Image and Graphics, 2002, vol. 4875, pp. 552-558.

[23] C. Harris, M. Stephens, "A combined corner and edge detection," Proc.of $4^{\text {th }}$ Alvey Vision Conf., 1988, pp. 147-151.

[24] F. Mindru, T. Tuytelaars, L. Van Gool, T. Moons, "Moment invariants for recognition under changing viewpoint and illumination," Computer Vision and Image Understanding, pp. 3-27, 2004.

[25] G. Carneiro, A. Jepson, "Multi-scale phase-based local features," IEEE Proc. of Computer Society Conf. on Computer Vision and Pattern Recognition, 2003, pp. 736-743.

[26] W. T. Freeman, E. H. Adelson, "The design and use of steerable filters," IEEE Transactions on Pattern Analysis and Machine Intelligence, vol. 1(3), pp. 891-906, 1991.

[27] Z. Feng, Q. M. Huang, H. Wang, W. Gao, "MOCC: A fast and robust correlation-based method for interest point matching under large scale changes," EURASIP Journal of Advanced Signal Processing, vol. 88, 2010.

[28] S. S. Tsai, D. Chen, G. Takacs, V. Chandrasekhar, R. Vedantham, R. Grzeszczuk, B. Girod, "Fast geometric re-ranking for image-based retrieval," Proc. of $17^{\text {th }}$ IEEE Int. Conf. on Image Processing, 2010, pp. 1029-1032.

[29] E. Herv, J. Egou, M. Douze, C. Schmid, "Hamming embedding and weak geometric consistency for large scale image search," Proc. of European Conf. on Computer Vision, pp 304-317, 2008. 
[30] J. Heinly, E. Dunn, J. M. Frahm, "Comparative Evaluation of Binary Features," European Conf. on Computer Vision, 2012. http://www.cs.unc.edu/ jheinly/feature-evaluation/datasets.html\#citeHeinly

[31] M. Chen, Z. Shao, D. Li, J. Liu, "Invariant matching method for different viewpoint angle images," Applied Optic., vol. 52(1), pp. 96-104, 2013. 